\title{
ANOMALIE MORFOLOGICHE IN ALCUNI PARADIGMI VERBALI DEL DIALETTO LOMBARDO DELLA BRIANZA NORD-ORIENTALE ${ }^{1}$
}

\author{
Fabio RIPAMONTI \\ Università della Boemia meridionale
}

\begin{abstract}
Riassunto (It): Il presente articolo è incentrato su una variante dialettale ancora poco studiata del lombardo occidentale che viene parlata nelle località attorno al Monte di Brianza (provincia di Lecco, Italia). Nella prima parte ne vengono dapprima delineate le coordinate storico-geografiche e le sue peculiarità rispetto alle varietà contigue, successivamente vengono analizzati tre punti della morfologia verbale di questo dialetto che mostrano un comportamento anomalo: 1) una serie di imperativi che terminano o per etimologia o per analogia con una velare e che attraverso l'aggiunta di un infisso -ar- possono esprimere una modalità a cavallo tra imperativo e futuro; 2) una distribuzione dell'infisso incoativo -(i/e)sk- che a causa dell'effetto del sincretismo mostra un forte legame con le parlate galloromanze; 3 ) un doppio modello di flessione del condizionale semplice in cui si possono osservare fenomeni di variabilità non solo morfologica. In tutti e tre $\mathrm{i}$ casi, l'analisi si è concentrata sull'interpretazione dei pochi dati finora a disposizione con l'obiettivo di collocare il brianzolo nell'ambito dell'attuale e proficua ricerca sui dialetti italiani (BENINCÀ et al., 2014) applicando ai temi in questione il quadro teorico dell'Autonomous Morphology (ARONOFF, 1994; MAIDEN et al., 2011; MAIDEN et al., 2013; MAIDEN, 2018).
\end{abstract}

\begin{abstract}
En): The present article focuses on a dialectal variant of Western Lombard that is spoken in the villages around the «Monte di Brianza» (district of Lecco, Italy) and which has been hardly studied. In the first section, historical-geographical coordinates and peculiarities of this dialect are outlined in relation to contiguous varieties. After that, three issues of the verbal morphology of brianzolo showing an anomalous behaviour are analysed: 1) a series of imperatives that end either by etymology or by analogy with a velar and that through the addition of an infix -ar-can express a modality between imperative and future; 2) a distribution of the inchoative infix -(i/e)sk- that, due to the effect of syncretism, shows a strong link to Gallo-romance dialects; 3 ) a double model of simple conditional flexion in which phenomena of variability can be observed, not only in morphological sense. In all three cases, the analysis focused on the interpretation of restricted data available by now with the aim of placing brianzolo within the current and profitable research on Italian dialects (BENINCÀ et al., 2014), applying to these instances the theoretical frame of Autonomous morphology (ARONOFF, 1994; MAIDEN et al., 2011; MAIDEN et al., 2013; MAIDEN, 2018).
\end{abstract}

Parole chiave (It): dialetto brianzolo; paradigma verbale romanzo; imperativo; condizionale; infisso incoativo -(i/e)sk-; dialetti italoromanzi; morfoma

Keywords (En): brianzolo dialect; Romance verbal paradigm; imperative; conditional; inchoative infix -(i/e)sk-; Italian dialects; morphome

Negli anni recenti la ricerca sulla morfologia dei paradigmi verbali romanzi ha visto un notevole sviluppo grazie a numerose prospettive, le più importanti delle

\footnotetext{
${ }^{1}$ Un ringraziamento particolare va ai miei informatori, in special modo ad Anselmo Luigi Brambilla, per la preziosa pazienza con cui hanno risposto alle mie pedanti richieste.Una versione preparatoria del presente articolo è stata discussa durante il 14th Cambridge Italian Dialect Syntax-Morphology Meeting (CIDSM14) tenutosi a Praga (27-29 maggio 2019) : vorrei esprimere la mia riconoscenza a tutti coloro che in questa occasione hanno contribuito con consigli,idee e spunti,in particolar modo Pavel Štichauer, Michele Loporcaro e Diego Pescarini, oltre agli anonimi recensori.
} 
quali sono la Autonomous Morphology (ARONOFF, 1994; MAIDEN et al., 2011; MAIDEN et al., 2013; MAIDEN, 2018), la Canonical Typology (CORBETT, 2005; 2016) e l'Ipotesi di Autonomia Paradigmatica (PIRRelli, 2000; PIRRELlBATTISTA, 2000). Ciò che accomuna queste linee di ricerca è un approccio orientato alla spiegazione del motivo per cui alcune presunte anomalie morfologiche riscontrabili in diverse lingue, $\mathrm{e}$ in modo ancor più evidente nelle varietà romanze, mostrino un comportamento coerente e per molti versi simile nonostante la loro distanza sia nello spazio che nel tempo. Se nel caso delle lingue romanze principali la descrizione delle irregolarità verbali pare ormai aver raggiunto un ottimale livello di completezza, perlomeno nelle sue linee principali, l'interesse per le parlate minori, e nello specifico per i dialetti italoromanzi, rappresenta un'ulteriore sfida per la verifica dei principi teorici applicati ai processi morfologici osservati in diacronia (VINCENT, 2014: 1-3). In questo senso, il presente articolo si pone un duplice scopo: introdurre la peculiarità di un dialetto circoscritto a una zona estremamente limitata e che finora non ha ricevuto particolari attenzioni, ossia il brianzolo delle località che si trovano attorno al cosiddetto «Monte di Brianza» (provincia di Lecco), e proporre un'applicazione delle teorie morfologiche di riferimento su alcuni aspetti anomali riscontrati nel verbo brianzolo, osservandoli in un complesso contesto di relazioni linguistiche col restante mondo romanzo.

\section{I limiti storico-geografici della Brianza}

La cosiddetta «Brianza $»^{2}$ è una regione storica della Lombardia amministrativa suddivisa in modo irregolare tra le attuali province di Lecco, Como e MonzaBrianza. I confini geografici sono stati spesso motivo di dibattito soprattutto nell'ambito del folklore e dell'etnografia; dal punto di vista linguistico Francesco CHERUBINI (1859: 289) proponeva una delimitazione piuttosto ristretta:

«È quindi comunemente considerato per Brianza tutto quel delizioso paese che ̀̀ conterminato dal fiume Lambro a ouest e dall'Adda a est, dal torrente Ravella e dalla Vallemadrera a nord, e da quella Via postale che da Canonica di Lambro mette a Vimercato e da questo borgo a Imbersago e Brivio lungo l'Adda.»

Una proposta di confini più ampia, e oggi comunemente accettata, sposta invece il limite occidentale della Brianza fino al fiume Seveso, mentre quello meridionale viene esteso fino a inglobare il territorio urbano di Monza, città che nonostante la posizione eccentrica rispetto al territorio brianzolo nel suo complesso e la vicinanza con un centro delle dimensioni di Milano costituisce ancora oggi il capoluogo riconosciuto della Brianza, considerazione giustificata linguisticamente anche dal

\footnotetext{
${ }^{2}$ Spesso l'etimologia del toponimo Brianza viene ancora discussa. La proposta più accreditata lo fa risalire al celtico brig 'altura, collina'. Altre proposte fanno riferimento a formulazioni non adeguatamente documentate, che collegano ad esempio il nome Brianza a un ipotetico Briganzia 'terra di briganti' oppure al nome Brianteo, comandante delle truppe di Belloveso, capo degli Insubri che scesero nel IV sec. a. C. in Italia stanziandosi tra la Svizzera orientale e l'attuale Lombardia e di cui parla Tito Livio nel capitolo V del I libro della sua opera $A b$ Urbe condita. Secondo altre opinioni alquanto discutibili non mancherebbero collegamenti toponomastici a testimonianza di un'unità etnico-culturale con altri territori europei (Briançon, Britannia, Bregenz...).
} 
fatto che la variante monzese presenta caratteristiche specifiche rispetto a quella milanese (BONFADINI, 2010a: 219). L'estensione del confine settentrionale della Brianza, invece, data la conformazione del territorio che va restringendosi a punta verso il lago di Como in direzione della città di Lecco, pare essere molto più elastico e va pian piano scemando nella percezione dei parlanti, che a seconda dei casi includono la città lariana nella Brianza o pongono il limite alle alture che prendono il nome di 'Triangolo di Canzo', leggermente più a sud di Lecco. Pare che l'espansione dei confini della Brianza sia stata dovuta al fatto che nel passato il territorio era un luogo di villeggiatura molto ambito dall'aristocrazia e dall'alta borghesia milanese; andare in Brianza per trascorrere un periodo di riposo era sinonimo di prestigio sociale che nel corso del tempo non tutti si potevano permettere, pertanto i limiti della regione tendevano ad avvicinarsi sempre di più alle zone di campagna vicine all'area metropolitana.

Fig. 1a, b, c-la posizione geografica della Brianza

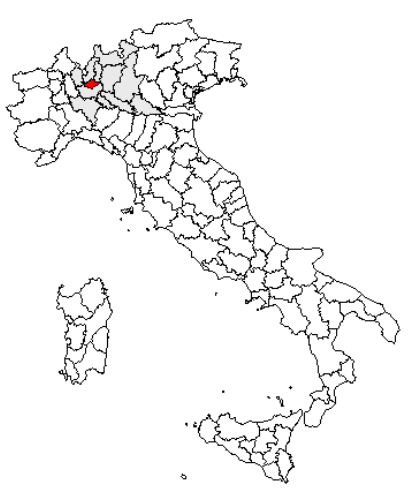

a

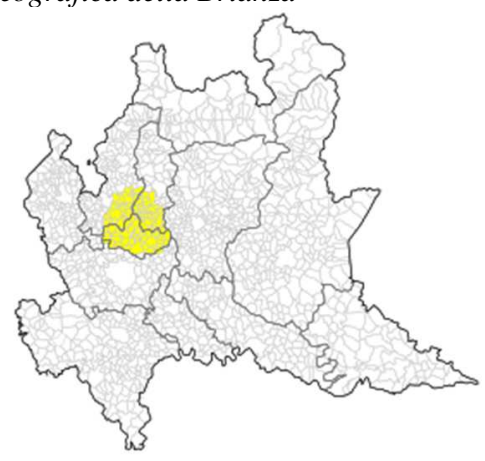

b

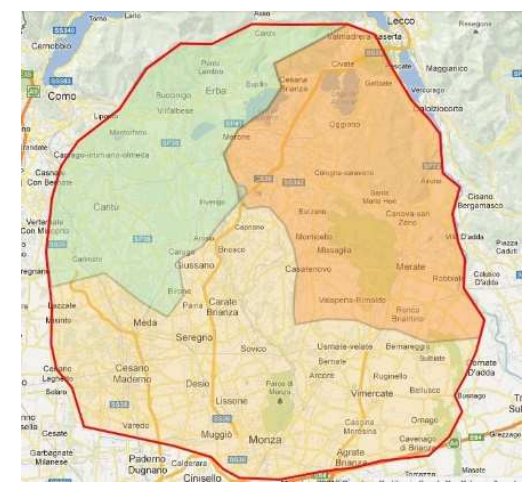

C 
Dal punto di vista storico, le prime testimonianze risalgono alla presenza di tribù celtiche preromane, in particolar modo gli Insubri ${ }^{3}$, che avevano occupato la zona a ovest del fiume Adda ed erano entrati in conflitto coi Cenomani, popolazione presente invece sulla sponda orientale. Questo forte confine geografico ed etnico venne rafforzato anche nel periodo della dominazione romana per via dell' organizzazione delle province a opera dell'imperatore Diocleziano (244-313), che aveva riportato il confine tra la X Regio Transpadana e la XI Regio Venetia et Histria dal fiume Oglio proprio all'Adda. In epoca moderna, a partire dal XV secolo questa separazione venne ribadita dalla frontiera tra il Ducato di Milano e la Repubblica di Venezia, perlomeno fino all'istituzione del Regno lombardo-veneto sotto il dominio austriaco (1814). L'occupazione da parte di altre popolazioni straniere altalenò periodi di unità a periodi di divisioni amministrative del territorio: nel medioevo la dominazione gotica, in seguito longobarda e franca, lasciarono tracce importanti nella lingua e nella cultura della Brianza, legando il suo percorso storico sostanzialmente a quello del resto dell'Italia centro-settentrionale. Nell'epoca moderna si alternarono il dominio dapprima francese, poi spagnolo e successivamente austriaco fino all'annessione della Lombardia al Regno di Sardegna (1859). ${ }^{4}$

La Brianza è sempre stata una zona molto popolata sia a causa della presenza di cospicue fonti d'acqua che per via della sua posizione strategica a ridosso delle Alpi. Il suo territorio era attraversato da diverse vie di comunicazione che mettevano in contatto il resto della penisola italiana con le zone al di là delle Alpi ma che si svilupparono anche lungo la direttrice est-ovest, sul percorso che grosso modo portava da Aquileia verso le Gallie. La ricerca in ambito archeologico sta cercando di identificare con precisione i tracciati di queste strade ricorrendo sovente anche alle ricche testimonianze toponomastiche: un esempio tra $i$ tanti si può osservare nel caso della via Ulteria, che partendo da Genova, punto di arrivo della via Aurelia, si orientava verso nord toccando Pavia, per poi proseguire lambendo a est Milano e dividersi in Brianza, nella località di Beolco, in due tronconi, uno che proseguiva costeggiando il percorso del fiume Adda fino al passo dello Spluga e oltre verso la città di Lindau (attualmente in Germania), un altro che deviava in direzione ovest verso le Gallie; i toponimi collegati in modo più o meno evidente con la via Ulteria (Sesto Ulteriano, Calolziocorte, Dolzago...) possono essere considerati tracce significative del suo itinerario (cfr. RIVA, 1987). ${ }^{5}$ Nella situazione descritta, di conseguenza, viene naturale aspettarsi che le varie limitazioni storiche, geografiche ed etniche delineate qui sopra non vadano interpretate in modo troppo restrittivo, ma che debbano essere inserite in un contesto molto più elastico, fatto di scambi e di contatti reciproci, anche linguistici, tra diverse popolazioni.

\footnotetext{
${ }^{3}$ Alle volte il lombardo occidentale viene definito come dialetto 'insubre' ma a causa delle implicazioni politiche del termine si preferisce in questa sede evitare interpretazioni in tal senso.

${ }^{4}$ Per un excursus sulla storia documentata della Brianza, cfr. http ://montedibrianza.it/?q=il-monte-dibrianza-nel-tempo [consultato il 28-8-2019]

${ }^{5}$ Attualmente sono in corso promettenti ricerche archeologiche su una via romana chiamata Longa, che da Como portava probabilmente verso la località di Brivio, sul fiume Adda, superato il quale si riconnetteva con altre strade romane verso i centri dell'Italia nordorientale.
} 


\section{Il dialetto del Monte di Brianza all'interno della variante lombarda occidentale}

\section{1. Le caratteristiche generali dei dialetti lombardi}

Il brianzolo parlato nella zona del cosiddetto Monte di Brianza si inserisce nella più ampia variante lombarda occidentale che ha storicamente in Milano il centro di maggior prestigio. La divisione tra un lombardo occidentale, considerato unitario grazie alla forza centripeta esercitata dal prestigio del milanese, e un lombardo orientale, privo di centri culturali altrettanto significativi, separati dal corso del fiume Adda, limite geografico che ribadiva la divisione di sostrato preromana, va attribuita a Bernardino BIONDELLI (1853) e nella sostanza viene mantenuta anche nei lavori degli autori successivi. L'unità linguistica lombarda doveva essere però molto più forte nel passato, almeno fino al medioevo, visto che alcuni fenomeni presenti in territori periferici (Trentino, Comasco, zone rurali della Brianza) concordano tra di loro (BONFADINI, 2010b: 840). In questa prospettiva, quindi, la divisione tra un lombardo occidentale e uno orientale (o 'cisabduano' e 'transabduano', se si prende come punto di riferimento Milano), sebbene valida in linea di massima ancora oggi, va integrata nel caso del dialetto brianzolo con quanto specificato nel paragrafo precedente a proposito della sua specificità storicogeografica, che lo rende di conseguenza un dialetto sostanzialmente di transizione.

Tralasciando gli aspetti comuni anche ad altri dialetti settentrionali, come la lenizione delle occlusive sorde intervocaliche (cadena 'catena') e lo scempiamento delle consonanti geminate (gata 'gatta'), le parlate lombarde presentano caratteristiche peculiari che riguardano in particolar modo la fonetica e la fonologia: la presenza di una serie di vocali anteriori turbate (foeuc /' $f \emptyset k$ / 'fuoco', mür /'myr/ 'muro'); la caduta di tutte le vocali finali ad esclusione della 'a'; la velarizzazione del nesso /al/ davanti a consonante (olt 'alto', molta 'malta'). Per la morfologia del gruppo nominale vanno ricordati i plurali metafonetici del tipo chèl, chii 'quello, quelli', cavèl, cavii 'capello, capelli'; la caduta delle sillabe finali degli infiniti dei verbi di I e III coniugazione (cantà 'cantare', finì 'finire'). Alcuni elementi sintattici sottolineano in modo più marcato la comunanza dei dialetti lombardi col dominio galloromanzo, come la negazione postverbale (me voeuri no/minga/mia 'io non voglio'), parallelo al francese parlato contemporaneo (cfr. je veux pas) o al tedesco (cfr. Ich sprache nicht deutsch); il raddoppiamento obbligatorio dei pronomi soggetto per la $2^{\mathrm{a}} \mathrm{e} 3^{\mathrm{a}}$ persona singolare (me so 'io sono' Vs. ti te sèt, lü l'è 'tu sei, lui è'); la perifrasi continuativa del tipo se t'è dré a fò cus'è? (lett. 'cosa sei dietro a fare cosa?' = 'cosa stai facendo?', cfr. fr. qu'est-ce que tu es en train de faire?); la diffusione di verbi sintagmatici (trà via 'buttare', lett. 'tirare via') (LOPORCARO, 2009: 97-101; BONFADINI, 2010b: 223-224; BENINCÀ et al., 2016: 187-193).

Dal punto di vista della conformazione del vocabolario, può essere interessante notare come nei dialetti lombardi in genere e ancor di più nelle parlate maggiormente conservative come è tendenzialmente il brianzolo, siano presenti diversi fondi lessicali che testimoniano della stratificazione etnica avvenuta nella regione. Nello specifico della variante qui analizzata, a una base evidentemente latina, alle volte ancora più conservativa in confronto ad altre varietà italoromanze (lat. armeniacum > brianz. mugnaac 'albicocca', lat. persicum $>$ brianz. persec 
'pèsca', lat. nec guttam > brianz. negott 'niente'), si sono sovrapposti influssi dovuti con buona probabilità al contatto coi diversi adstrati germanici, sia in epoca medievale che in quella più recente della dominazione austriaca (lat. astrologus $>$ brianz. stroeulec 'zingaro', cfr. ted. Strolch 'vagabondo'; got. blauths > brianz. biott 'nudo'; long. strak > brianz. strac 'stanco'; brianz. bauscia 'pallone gonfiato', cfr. ted. bauschen 'gonfiare' $)^{6}$, oppure molto probabilmente dovuti alla presenza francese e spagnola nel territorio nelle diverse fasi storiche (brianz. tumat 'pomodoro', cfr. fr. sp. tomate; brianz. incoeu 'oggi', cfr. occ. encuei, ancuei; brianz. serà, cfr. sp. cerrar; brianz. loeuch 'pazzo, stupido', cfr. sp. loco; brianz. giambòn 'prosciutto', cfr. fr. jambon, sp. jamon). Alcuni altri termini di media frequenza sembrano perlopiù delle innovazioni interne, considerandone l' assenza in altre lingue (magiuster 'fragola' = frutto di maggio?; butásc 'pancia gonfia'), benché in alcuni casi vi si possa ravvisare un legame con un fondo probabilmente prelatino esteso anche al territorio d'oltralpe di lingua romancia (crapa 'testa dura, modo di pensare ostinato', cfr. romancio crap 'masso di pietra').

\section{2. Le specificità del dialetto brianzolo rispetto alla varietà lombarda orientale e a quella milanese}

Il dialetto brianzolo presenta alcune caratteristiche che lo distinguono dalle parlate contigue. Le diversità con il lombardo orientale si basano soprattutto su alcune peculiarità fonetiche e fonologiche. Il tratto distintivo più marcato riguarda il valore fonologico della lunghezza vocalica, presente a occidente ma assente a oriente (lomb. occ. me so naa 'io sono andato' Vs. me voeuri mia nà 'io non voglio andare'). Inoltre, le due varianti differiscono nella realizzazione $\mathrm{di} / \mathrm{n} /$ finale (conservata a occidente tranne che davanti a /e/, dove cade allungando la vocale stessa, anche solo come nasalizzazione della vocale precedente, come ad esempio in man, can, vin vs. $m a, c a, v i$ 'mano, cane, vino', mentre scompare a oriente) e di $/ \mathrm{v} /$ in posizione intervocalica (conservata a occidente e caduta oppure spirantizzata a oriente, come caval vs. ca'hal 'cavallo'). Naturalmente anche nel lessico si possono trovare esempi distintivi, come il caso di bagaj Vs. s-cèt 'ragazzo'; scighéra Vs. geba 'nebbia'.

I tratti distintivi del brianzolo rispetto alla varietà milanese sono meno marcati in confronto a quelli che lo differenziano dal lombardo orientale per via soprattutto della forza irradiatrice che Milano ha sempre esercitato su buona parte della regione come centro politico e in particolar modo religioso grazie alla potenza della sua arcidiocesi. Dal punto di vista fonetico colpisce la velarizzazione della /a/ in sillaba tonica davanti a nasale (fòm 'fame', còn 'cane', biònca 'bianca'), che si ritrova anche negli infiniti della I coniugazione (magnò 'mangiare', cumandò 'comandare') $\mathrm{e}$ in alcuni monosillabi (cò 'casa', sciò 'qui', giò 'giù'). Un altro fenomeno caratteristico è il rafforzamento di /s/ sorda dopo consonante fino al grado di affricata dentale (falz 'falso', inverz 'inverso'), che si manifesta anche in fonetica sintattica (ul Zignùr 'il Signore', me'l zo mia 'io non lo so') (BONFADINI, 2010a: 227-231). Nel lessico, tenendo presente la posizione periferica del brianzolo rispetto al milanese, e di conseguenza una più probabile arcaicità nel vocabolario, si possono

${ }^{6}$ https ://patrimonilinguistici.it/dieci-parole-lombarde-origine-germanica/ [consultato il 28-9-2019] - 36 - 
ricordare alcuni termini legati alla vita quotidiana, come zibret Vs. sciavat 'ciabatta', gnef vs. carotul 'carota', evidentemente meno italianizzati rispetto ai corrispettivi milanesi e presenti anche nei dialetti lombardi del Canton Ticino (cfr. Lessico dialettale della Svizzera italiana, Bellinzona, Centro di dialettologia e di etnografia, 5 volumi, 2004).

Se da un lato il brianzolo appartiene pienamente alla variante lombarda occidentale, da un altro punto di vista è appurata la presenza di tratti risalenti con buona probabilità agli scambi intrattenuti con i territori della sponda orientale dell'Adda. Anche dal punto di vista più prettamente geografico, lungo il letto del fiume che dalla città di Lecco scorre in direzione sud-est come emissario del lago di Como, in alcuni punti i due territori non sono così nettamente separati, perlomeno finché il paesaggio cambia aspetto all'incirca all'altezza delle località di Porto e di Trezzo d'Adda, divenendo molto più impetuoso nel suo percorso e, in riferimento al passato, rendendo probabilmente meno agevole l'attraversamento del fiume in queste zone. L'aspetto linguistico più evidente che caratterizza i dialetti lombardi orientali e che si ritrova nel brianzolo è l' abbassamento delle vocali chiuse anteriori /i/ e /y/ toniche rispettivamente a /e/ chiusa e a / $\varnothing /$ se la vocale si trova in sillaba finale (spina 'spina' vs. spén 'spine'; brüta 'brutta' Vs. bröt 'brutto', vün 'uno' Vs. vöna 'una'), fenomeno che nelle varianti brianzole della zona lecchese viene generalizzato a tutte le vocali alte brevi toniche (rùssa 'rossa' vs. rós 'rosso'; véna 'vena' vs. vèn 'vene'; buna 'buona' Vs. bón 'buono'; séca 'secca' Vs. sèc 'secco'). Un altro fenomeno comune è il maschile con uscita consonantica dei sostantivi di genere femminile ('donna' la dona, i don; 'gamba' la gomba, i gomb; 'ragazza' la tusa, i tuson).

I problemi legati all'analisi in diacronia del dialetto brianzolo riguardano soprattutto le tardive documentazioni scritte, poiché il primo testo sicuramente riconducibile a questa parlata è presente nella raccolta di Giovanni Papanti I parlari italiani in Certaldo del 1875, in cui si trova la traduzione di alcune righe della novella I, 9 del Decamerone di Giovanni Boccaccio nel dialetto della località di Missagliola; Gianfranco Ravasi avrebbe però individuato alcune parole brianzole nella produzione di Carlo Maria Maggi, autore milanese del XVII secolo, aventi come scopo la rappresentazione realistica dei dialoghi tra personaggi di città e di campagna ${ }^{7}$. Inoltre, le indagini sul campo effettuate finora sono ancora piuttosto disomogenee e riservate a uno scarso numero di località (BONFADINI, 2010a: 220221), ciò dovuto probabilmente anche al fatto che una vera e propria delimitazione del dialetto brianzolo rispetto a quello milanese è un risultato relativamente recente, poiché a partire dalle famose note del CHERUBINI (1856) non si è verificato, se non negli anni recenti, un interesse che non fosse legato agli aspetti folkloristici (cfr. THOMPSON, 2015).

\section{Anomalie morfologiche riscontrate nel dialetto del Monte di Brianza}

Sulla base dei dati finora a disposizione, che però necessitano ancora di un trattamento approfondito in riferimento a quanto finora esposto, nella variante del

\footnotetext{
${ }^{7}$ https ://www.wikiwand.com/it/Dialetto_brianzolo [consultato il 28-9-2019]
} 
brianzolo oggetto di studio sono stati riscontrati alcuni fenomeni che paiono deviare, almeno parzialmente, in modo del tutto specifico dalle aspettative relative ai paradigmi verbali di una parlata romanza, anche nel caso di verbi irregolari. I fenomeni riscontrati sono: 1) la presenza di una velare non etimologica nell'imperativo dei verbi dò 'dare' e stò 'stare' per i quali è stata anche riscontrata una probabile strategia morfologica che permette una sorta di imperativo futuro limitato a questi verbi e ai verbi riflessivi (3. 1. ); 2) la distribuzione dell'infisso incoativo -(i/e)sk- nei paradigmi di alcuni verbi derivanti dalla IV coniugazione latina (3. 2); 3) una doppia serie di condizionale semplice del tipo vegnaría/vegnarés, entrambi col significato di 'io verrei', il secondo dei quali pone delle questioni sulla sua origine e sulle specificità d'uso (3. 3).

\section{1. La presenza di una velare non etimologica nell'imperativo dei verbi do 'dare' e sto 'stare' e una probabile forma di imperativo futuro}

Negli studi tradizionali di morfologia romanza, il modo imperativo non si è riservato una particolare attenzione nelle trattazioni teoriche, tuttavia la sua centralità è stata riconsiderata sulla base del fatto che i dati provenienti dalle ricerche sull'acquisizione linguistica a quanto pare dimostrerebbero che le forme dell'imperativo sono tra le prime a essere interiorizzate dai bambini (cfr. MAIDEN et al., 2009: 106 con ulteriori riferimenti bibliografici). Inoltre, la mole di dati sull'imperativo provenienti dai dialetti italoromanzi sta portando a galla alcuni fenomeni di estremo interesse che hanno iniziato ad attirare l'attenzione degli esperti negli anni recenti, sebbene manchi ancora una trattazione sistematica sul tema (cfr. MAIDEN et al., 2009; MAIDEN, 2010; SwEARING, 2011; FlORICIC ET MOLINU, 2012).

Come ben noto, in latino esistevano due tipi di imperativo, presente e futuro, distinti dal punto di vista degli esponenti morfologici non solo rispetto all'indicativo, a differenza di alcune lingue romanze come l'italiano, il francese o il rumeno, ma anche all'interno dei propri paradigmi, poiché la persona, il numero o la classe verbale di appartenenza venivano segnalati in modo piuttosto trasparente:

Tab. 1 - Coniugazione regolare dell'imperativo latino

\begin{tabular}{|c|c|c|c|c|c|}
\hline & & I. laudare & II. monēre & III. legěre & IV. audire \\
\hline \multirow[t]{2}{*}{ Presente } & $2 \mathrm{sg}$. & laudā & monē & legě & audī \\
\hline & $2 \mathrm{pl}$. & laudāte & monēte & legĭte & audīte \\
\hline \multirow[t]{4}{*}{ Futuro } & $2 \mathrm{sg}$. & laudāto & monēto & legĭto & audīto \\
\hline & 3 sg. & laudāto & monēto & legĭto & audìto \\
\hline & $2 \mathrm{pl}$. & laudatōte & monetōte & legitōte & auditōte \\
\hline & $3 \mathrm{pl}$. & laudānto & monēnto & legūnto & audiūnto \\
\hline
\end{tabular}

Solo in quattro casi l'imperativo latino presentava delle forme monosillabiche con un' uscita consonantica, ossia nella $2^{\text {a }}$ pers. sg. dei verbi dicere 'dire' (dic), ducere 'condurre' (duc), facere 'fare' (fac) e ferre 'portare' (fer) (tab. 2): 
Tab. 2 - Coniugazione irregolare dell'imperativo in latino

\begin{tabular}{|l|l|l|l|l|l|}
\hline \multicolumn{2}{|c}{} & dicere & ducere & facere & ferre \\
\hline \multirow{3}{*}{ Presente } & $2 \mathrm{sg}$. & dic & duc & fac & fer \\
\cline { 2 - 6 } & $2 \mathrm{pl}$. & dicite & ducite & facite & ferte \\
\hline \multirow{5}{*}{ Futuro } & $2 \mathrm{sg}$. & dicito & ducito & facito & ferto \\
\cline { 2 - 6 } & $3 . \mathrm{sg}$. & dicito & ducito & facito & ferto \\
\cline { 2 - 6 } & $2 \mathrm{pl}$. & dicitote & ducitote & facitote & fertote \\
\cline { 2 - 6 } & $3 \mathrm{pl}$. & dicunto & ducunto & faciunto & ferunto \\
\hline
\end{tabular}

Nella $2^{\text {a }}$ pers. sg. dell'imperativo del verbo dé 'dire' il brianzolo ha conservato la velare dell'originale latino (lat. $d i c>$ brianz. $d e c$ ), la quale si è estesa poi analogicamente anche alla $1^{\mathrm{a}}$ e alla $2^{\mathrm{a}}$ pers. pl. (tab. 3). La velare non compare in altre celle del paradigma di questo verbo, all'interno del quale la base si è livellata sugli esiti tipici per i dialetti lombardi di $/ \mathrm{k} /+$ vocale palatale $>/ \mathrm{s} /$, fenomeno di conguaglio tematico ben attestato anche in molti altri dialetti italoromanzi (ROHLFS, 1968: 263):

Tab. 3 - Presente indicativo e imperativo di dé 'dire'8

\begin{tabular}{|l|l|l|l|l|l|l|}
\hline & $1 \mathrm{sg}$ & $2 \mathrm{sg}$ & $3 \mathrm{sg}$ & $1 \mathrm{pl}$ & $2 \mathrm{pl}$ & $3 \mathrm{pl}$ \\
\hline $\begin{array}{l}\text { Presente } \\
\text { Indicativo }\end{array}$ & disi & diset & dees & disèm & disìi & disen \\
\hline Imperativo & & dec & & disèmec & disìic & \\
\hline
\end{tabular}

Anche altri due verbi brianzoli che nel dominio italoromanzo spesso si influenzano reciprocamente, do 'dare' (tab. 4) e sto 'stare' (tab. 5), presentano un' uscita con velare nelle forme dell'imperativo, senza che però in questo caso vi possa essere un'apparente motivazione intrinseca della sua comparsa se non rifacendosi a un effetto, sempre di tipo analogico, dell'imperativo di dicere:

Tab. 4 - Presente indicativo e imperativo di dò 'dare'

\begin{tabular}{|l|l|l|l|l|l|l|}
\hline & $1 \mathrm{sg}$ & $2 \mathrm{sg}$ & $3 \mathrm{sg}$ & $1 \mathrm{pl}$ & $2 \mathrm{pl}$ & $3 \mathrm{pl}$ \\
\hline $\begin{array}{l}\text { Presente } \\
\text { Indicativo }\end{array}$ & do & dee & do & dem & dii & don \\
\hline Imperativo & & dac & & dèmec & diic & \\
\hline
\end{tabular}

Tab. 5 - Presente indicativo e imperativo di stò 'stare'

\begin{tabular}{|l|l|l|l|l|l|l|}
\hline & $1 \mathrm{sg}$ & $2 \mathrm{sg}$ & $3 \mathrm{sg}$ & $1 \mathrm{pl}$ & $2 \mathrm{pl}$ & $3 \mathrm{pl}$ \\
\hline $\begin{array}{l}\text { Presente } \\
\text { Indicativo }\end{array}$ & sto & stee & sto & stem & stii & ston \\
\hline Imperativo & & stac & & stèmec & stiic & \\
\hline
\end{tabular}

${ }^{8}$ La trascrizione dei dati relativi al brianzolo si limita agli interventi diacritici necessari per rappresentare i tratti significativi della pronuncia e non ha alcuna pretesa esaustiva in considerazione dell'ancora non unificata resa grafica soprattutto delle vocali turbate. 
La comparsa di una velare non etimologica non è un fenomeno anomalo nelle parlate romanze, in particolar modo nel dominio iberoromanzo (O'NEILL 2018). Oltre che all'imperativo dei verbi sopra indicati, in brianzolo una velare compare in enclisi nell'infinito avech 'avere', mentre nelle forme flesse dello stesso paradigma la velare diventa proclitica, (cfr.: gh'oo io ho', gh'avevi 'io avevo', gh'avaroo 'avrò', gh'avaria/gh'avarès 'io avrei'...) (CROLA, 2005: 119-121) probabilmente assimilando l'avverbio di luogo ghe 'ci' (ROHLFS, 1968: 275-276). In altre lingue romanze l'inserto velare viene spesso esteso ad altre celle di un paradigma verbale, non di rado seguendo un modello di tipo 'morfomico' (MAIDEN, 2011: 236-7): in catalano nel verbo tener la velare della $1^{\text {a }}$ pers. sg. del presente indicativo tinc si estende secondo un L-pattern fino a tutto il preterito; in linguadociano la base tengdello stesso verbo è estesa a tutte le persone del congiuntivo e del perfetto indicativo, oltre che al gerundio e al participio; numerosissimi dialetti italoromanzi ricorrono a un inserto velare non etimologico soprattutto nella $1^{\mathrm{a}}$ pers. sing. (lucano sugo 'io sono'; cilentano mecco 'io metto'; maceratese vaco 'io vado'). ${ }^{9}$

L'uscita consonantica come marca caratteristica degli imperativi brianzoli si può trovare anche nei verbi riflessivi:

Tab. 6-Presente indicativo e imperativo di se moeuvé 'muoversi'

\begin{tabular}{|l|l|l|l|l|l|l|}
\hline & $1 \mathrm{sg}$ & $2 \mathrm{sg}$ & $3 \mathrm{sg}$ & $1 \mathrm{pl}$ & $2 \mathrm{pl}$ & $3 \mathrm{pl}$ \\
\hline $\begin{array}{l}\text { Presente } \\
\text { Indicativo }\end{array}$ & $\begin{array}{l}\text { me } \\
\text { moeuvi }\end{array}$ & $\begin{array}{l}\text { te se } \\
\text { moeuvet }\end{array}$ & $\begin{array}{l}\text { el se } \\
\text { moeuve }\end{array}$ & se moeuvem & $\begin{array}{l}\text { se } \\
\text { moeuvìi }\end{array}$ & $\begin{array}{l}\text { se } \\
\text { moeuven }\end{array}$ \\
\hline Imperativo & & moeuves & & moeuvèmes & muvìis & \\
\hline
\end{tabular}

Un fenomeno particolare che accomuna sia i verbi aventi un imperativo con un'uscita velare che quelli riflessivi è l'esistenza di forme morfologicamente a cavallo tra quelle dell'imperativo e del futuro (oppure interpretabili con l'inserimento di un infisso -ar-) che, a quanto pare, esprimerebbero una sorta di imperativo futuro, tempo completamente scomparso nel passaggio dal latino alle lingue romanze senza aver lasciato alcuna traccia conosciuta in alcuna variante (tab. 7):

Tab. 7 - Futuro semplice e imperativo futuro [?] di dò 'dare', stò 'stare, se moeuvé 'muoversi'

\begin{tabular}{|l|l|l|l|l|l|l|}
\hline & $1 \mathrm{sg}$ & $2 \mathrm{sg}$ & $3 \mathrm{sg}$ & $1 \mathrm{pl}$ & $2 \mathrm{pl}$ & $3 \mathrm{pl}$ \\
\hline Futuro semplice & daroo & daree & daroo & darèm & darìi & daròn \\
\hline $\begin{array}{l}\text { Imperativo } \\
\text { futuro [?] }\end{array}$ & & darec & & darèmec & darìic & \\
\hline
\end{tabular}

\begin{tabular}{|l|l|l|l|l|l|l|}
\hline & $1 \mathrm{sg}$ & $2 \mathrm{sg}$ & $3 \mathrm{sg}$ & $1 \mathrm{pl}$ & $2 \mathrm{pl}$ & $3 \mathrm{pl}$ \\
\hline Futuro semplice & staroo & staree & staroo & starèm & starìi & staròn \\
\hline $\begin{array}{l}\text { Imperativo } \\
\text { futuro [?] }\end{array}$ & & starec & & starèmec & starìic & \\
\hline
\end{tabular}

\footnotetext{
${ }^{9}$ Gli esempi sono tratti nella quasi totalità dal database http ://romverbmorph.clp.ox.ac.uk/ [consultato
} il 28-9-2019] 


\begin{tabular}{|l|l|l|l|l|l|l|}
\hline & $1 \mathrm{sg}$ & $2 \mathrm{sg}$ & $3 \mathrm{sg}$ & $1 \mathrm{pl}$ & $2 \mathrm{pl}$ & $3 \mathrm{pl}$ \\
\hline $\begin{array}{l}\text { Futuro } \\
\text { sem- } \\
\text { plice }\end{array}$ & $\begin{array}{l}\text { me } \\
\text { moeuvaroo }\end{array}$ & $\begin{array}{l}\text { te se } \\
\text { moeuvaret }\end{array}$ & $\begin{array}{l}\text { el se } \\
\text { moeuvaroo }\end{array}$ & $\begin{array}{l}\text { se } \\
\text { moeuvarèm }\end{array}$ & $\begin{array}{l}\text { se } \\
\text { moeuvarìi }\end{array}$ & $\begin{array}{l}\text { se } \\
\text { moeuvaròn }\end{array}$ \\
\hline $\begin{array}{l}\text { Impera- } \\
\text { tivo } \\
\text { futuro } \\
{[?]}\end{array}$ & & moeuvares & & $\begin{array}{l}\text { moeuva- } \\
\text { rèmes }\end{array}$ & muvarìis & \\
\hline
\end{tabular}

La distinzione sia morfologica che funzionale di queste forme da un imperativo e da un futuro pieni è ben presente nella consapevolezza dei parlanti, come si può dedurre dagli esempi (1) e (2), dunque non si possono interpretare come tipologie alternative di questi tempi né come forme aventi, ad esempio, un clitico pronominale o avverbiale, come testimoniato in altre varianti italoromanze (MAIDEN, 2007: 156157):

(1) Giuòn e Carletu, dariic vergot a stu poeur bagaj!

Giovanni e Carlino, 'dare' -IMP. FUT?. 2PL qualcosa a questo povero ragazzo!

'Giovanni e Carlino, date (più tardi) qualcosa a questo povero ragazzo!'

(2) Adès moeuvares mia, se ti te voeuret mia ciapà vüna pesciada!

Adesso 'muoversi'-IMP. FUT?. 2SG. NEG., se non vuoi prenderti un calcio!

'Adesso non muoverti (per un po'), se non vuoi prenderti un calcio!'

A conferma dell'esistenza di questo processo morfologico si può anche osservare che esso non può comparire negli imperativi di altri verbi che non prevedono l'uscita consonantica dell'imperativo, regolari o irregolari che siano (34):

(3) Maia pion!

*Maiara pion!

'Mangiare' - IMP. 2PL. SING. piano

'Mangia piano!'

(4) Vé sciò!

*Varé sciò!

'Venire' - IMP. 2PL. SING. qua

'Vieni qua!'

Un'ipotesi che in questa fase dell'osservazione dei dati qui presentati potrebbe essere formulata senza incorrere in errate valutazioni è che siamo difronte, più che a un residuo «archeologico» dell'imperativo futuro latino, a un'innovazione interna del brianzolo. La pressoché assoluta mancanza di tracce simili in altre parlate romanze (vd. Conclusioni), sia dal punto di vista più strettamente morfologico che da quello funzionale, è un elemento molto eloquente che spinge verso questa opinione. Inoltre, allo stato attuale della ricerca, non vi è alcun motivo logico in base al quale il brianzolo, che per quanto sia un dialetto conservativo nell'ambito del 
lombardo occidentale ha pur sempre mantenuto dal punto di vista della sua storia una posizione di dialetto di contatto, avrebbe dovuto conservare un tempo verbale assente persino nelle lingue romanze solitamente considerate più conservative, come il rumeno o il sardo. Molto più probabile potrebbe essere invece una spiegazione da ricercare nella complessità e nella varietà tipica per la morfologia verbale romanza quando deve esprimere il concetto di 'futuro', tempo di per sé tendente a rappresentare l'insicurezza e l'incertezza dei parlanti e campo aperto ad ogni genere di aspettative e di modalità, come il senso di dovere, di volontà o di obbligatorietà.

\section{2. La distribuzione dell'infisso incoativo $-(i / e) s k$ - in alcuni verbi della IV coniugazione}

Nel passaggio dal latino alle lingue romanze, come ben noto, l'infisso -(i/e)skha perso il suo originale valore incoativo rimanendo un morfema sciolto da vincoli grammaticali e privo del significato originale (cfr. lat. amo 'sono innamorato, amo' vs. amasco 'mi innamoro'). In seguito alla perdita della sua funzione, la distribuzione di questo aumento si presenta alquanto disomogenea nelle varie parlate romanze e pone delle questioni sul suo comportamento che hanno prodotto una corposa bibliografia; vi sono infatti lingue, come il logudorese e le lingue iberoromanze, che hanno esteso l'infisso a tutto il paradigma di alcuni verbi, altre come l'italiano e il rumeno che hanno optato per una distribuzione secondo il morfoma $N$-pattern (per il concetto e il dibattito relativo, cfr. ARONOFF, 1994; MAIDEN, 2018) mentre altre ancora lo hanno del tutto ignorato (per una casistica della distribuzione 'morfomica' dell'infisso e per una sintesi del tema, cfr. RIPAMONTI, 2017: 82-87, 90-97). Queste differenze rendono difficoltosa una spiegazione generale, valida per tutte le lingue romanze, di una qualsivoglia logica alla base della distribuzione dell'infisso incoativo, tanto più che esso manca sistematicamente in alcune celle dei paradigmi, come nel participio passato $o$ nell'infinito, mentre $\mathrm{i}$ dati provenienti dai dialetti galloromanzi mostrano la tendenza e estenderlo verso le basi del futuro e del condizionale (cfr. MEUL, 2010: 19-35).

In brianzolo, così come in italiano standard, l'infisso incoativo compare nel presente indicativo di alcuni verbi della IV coniugazione (tab. 8), anche in quelli, come se stufé, che pare abbiano subito un metaplasma dalla I alla IV:

(a)

Tab. 8 - Presente indicativo di capé 'capire' (a), parté 'partire' (b), se stufé (c) 'stufarsi'

\begin{tabular}{|l|l|l|l|l|l|}
\hline $1 \mathrm{sg}$ & $2 \mathrm{sg}$ & $3 \mathrm{sg}$ & $1 \mathrm{pl}$ & $2 \mathrm{pl}$ & $3 \mathrm{pl}$ \\
\hline capissi & capisset & capès & capissem & capìi & capissen \\
\hline
\end{tabular}

(b)

\begin{tabular}{|l|l|l|l|l|l|}
\hline $1 \mathrm{sg}$ & $2 \mathrm{sg}$ & $3 \mathrm{sg}$ & $1 \mathrm{pl}$ & $2 \mathrm{pl}$ & $3 \mathrm{pl}$ \\
\hline partissi & partisset & partès & partissem & partìi & partissen \\
\hline
\end{tabular}


(c)

\begin{tabular}{|l|l|l|l|l|l|}
\hline $1 \mathrm{sg}$ & $2 \mathrm{sg}$ & $3 \mathrm{sg}$ & $1 \mathrm{pl}$ & $2 \mathrm{pl}$ & $3 \mathrm{pl}$ \\
\hline me stüfissi & te se stüfisset & el se stüfès & se stüfìssem & se stüfii & se stüfissen \\
\hline
\end{tabular}

Come si può notare negli esempi nelle tabelle, l'infisso incoativo non compare sistematicamente nella $2^{\mathrm{a}}$ pers. pl. La particolarità di questa cella del paradigma si può osservare anche in un altro verbo della IV coniugazione come durmé 'dormire' (tab. 9), dove la vocale della base si innalza restringendosi fino a /u/, e nel fatto che l'accento, in contrasto con le altre forme, cade non sulla radice del verbo, bensì sulla desinenza, fenomeno sistematico nei verbi brianzoli:

Tab. 9- Presente indicativo di durmé 'dormire'

\begin{tabular}{|l|l|l|l|l|l|}
\hline $1 \mathrm{sg}$ & $2 \mathrm{sg}$ & $3 \mathrm{sg}$ & $1 \mathrm{pl}$ & $2 \mathrm{pl}$ & $3 \mathrm{pl}$ \\
\hline dormi & dormet & dorma & dormem & durmì & dormen \\
\hline
\end{tabular}

Paradigmi che presentano una particolarità nella cella della $2^{\mathrm{a}}$ pers. pl. non sono molto frequenti nelle lingue romanze, ma si possono ritrovare ad esempio nel portoghese ir 'andare', oppure rimanendo tra i dialetti lombardi nel monzese andà 'andare' (BENINCÀ et al., 2016: 194) o nel cremonese finì 'finire' (RosSINI, 1975) (tab. 10):

(a)

Tab. 10 - Presente indicativo del portoghese ir 'andare' (a), del monzese andà 'andare' (b) e del cremonese finì 'finire' (c).

\begin{tabular}{|l|l|l|l|l|l|}
\hline $1 \mathrm{sg}$ & $2 \mathrm{sg}$ & $3 \mathrm{sg}$ & $1 \mathrm{pl}$ & $2 \mathrm{pl}$ & $3 \mathrm{pl}$ \\
\hline vou & vais & vai & vamos & ides & vão \\
\hline
\end{tabular}

(b)

\begin{tabular}{|l|l|l|l|l|l|}
\hline $1 \mathrm{sg}$ & $2 \mathrm{sg}$ & $3 \mathrm{sg}$ & $1 \mathrm{pl}$ & $2 \mathrm{pl}$ & $3 \mathrm{pl}$ \\
\hline vo & vet & va & vem & andé & van \\
\hline
\end{tabular}

(c)

\begin{tabular}{|l|l|l|l|l|l|}
\hline $1 \mathrm{sg}$ & $2 \mathrm{sg}$ & $3 \mathrm{sg}$ & $1 \mathrm{pl}$ & $2 \mathrm{pl}$ & $3 \mathrm{pl}$ \\
\hline finissi & finisset & finis & finissum & finìi & finis \\
\hline
\end{tabular}

Un curioso parallelismo nell' italiano standard riguarda il verbo dire, in cui a differenza delle altre persone nella $2^{\mathrm{a}} \mathrm{pl}$. del presente indicativo non compare una sillaba contenente una velare $\mathrm{o}$ un' affricata, che però per analogia può manifestarsi nell'italiano regionale o sub-standard (tab. 11): 
Tab. 11 - Presente indicativo dell'italiano standard dire

\begin{tabular}{|l|l|l|l|l|l|}
\hline $1 \mathrm{sg}$ & $2 \mathrm{sg}$ & $3 \mathrm{sg}$ & $1 \mathrm{pl}$ & $2 \mathrm{pl}$ & $3 \mathrm{pl}$ \\
\hline dico & dici & dice & diciamo & $\begin{array}{l}\text { dite } \\
\text { (sub-standard } \\
\text { dicete) }\end{array}$ & dicono \\
\hline
\end{tabular}

A mio avviso, per interpretare questa particolare distribuzione, è necessario ricorrere a un approccio articolato che tenga presente diversi principi teorici, che presi nel loro insieme possono con buona probabilità contribuire a dar conto di una struttura verbale a quanto pare coerente e ben presente nella coscienza dei parlanti. Un primo concetto che viene in aiuto è ciò che Greville CORBETT propone sotto la definizione di 'morphomic split' (2016): con questa definizione si intende qualsiasi «partizione» di tipo morfologico all'interno di un paradigma, verbale ma non solo, non motivata da nessun parametro morfosintattico, come persona, numero o genere, o extralinguistico. In questo senso, dunque, il concetto di morfoma andrebbe applicato in questo caso in una delle sue accezioni «in negativo», ossia come una sorta di «ultima spiaggia» nel formalizzare una situazione altrimenti inspiegabile. Tuttavia l'evidenza e la diffusione dei dati riportati in questo paragrafo spinge a pensare che una struttura paradigmatica in cui la $2^{a}$ pers. pl. contrappone un esponente morfologico a quello delle altre celle del paradigma non sia un caso isolato o un' eccezione che non può trovare una descrizione formale. Sulla scia di quanto proposto da CORBETT, in questa conformazione potrebbero quindi intervenire anche altri principi morfologici spesso chiamati in causa nell'analisi dei paradigmi verbali, come il concetto di blocking, il principio di Pāninini o quello di Privileged Category Restriction (ARONOFF, 1976; STUMP, 2006; CORBETT, 2016), che potrebbero mettere in luce il motivo per cui proprio la cella della $2^{\mathrm{a}}$ pers. pl. venga esclusa dai fenomeni che interessano un paradigma verbale. A questo proposito, una prospettiva che andrebbe osservata sul campo è probabilmente quella legata al ruolo della frequenza e dell' acquisizione della morfologia verbale da parte dei bambini (BYBEE, 2007), poiché a quanto pare la $2^{\mathrm{a}}$ pers. pl. sarebbe la cella meno frequentemente utilizzata di un paradigma e anche l'ultima a essere imparata.

Molto più pertinente all' argomento è invece quanto esposto da HINZELIN (2011: 297-8) nella sua analisi del sincretismo nel dominio galloromanzo. Questo autore osserva che in lorenese alcuni verbi come naler 'andare' (tab. 12) nel presente indicativo hanno un paradigma in cui la $2^{\mathrm{a}}$ pers. pl. rimane isolata rispetto alle restanti celle, esattamente come i verbi brianzoli qui analizzati:

Tab. 12 - Presente indicativo di naler 'andare' in lorenese (Petit Rombach)

\begin{tabular}{|l|l|l|l|l|l|}
\hline $1 \mathrm{sg}$ & $2 \mathrm{sg}$ & $3 \mathrm{sg}$ & $1 \mathrm{pl}$ & $2 \mathrm{pl}$ & $3 \mathrm{pl}$ \\
\hline ve: & ve: & ve: & vã & al $\varepsilon:$ & vã \\
\hline
\end{tabular}

Secondo HiNZELIN, si tratta di un processo sincretico piuttosto costante e diffuso in galloromanzo in base al quale una cella tende a assorbirne un'altra riorganizzando il paradigma secondo un nuovo tipo di allomorfia. Nel caso del lorenese naler si sarebbe verificata un'attrazione nella propria orbita morfologica da parte della $3^{\text {a }}$ pers. pl. a scapito della $1^{\text {a }}$ pers. pl., intaccando di fatto l'originaria distribuzione 
suppletiva del tipo $N$-pattern tra i lessemi di ambulare e di vaděre. Tracce di questa distribuzione vengono segnalate anche in Bonvesin de la Riva (tab. 13), autore milanese medievale (DOMOKOS, 2007: 263):

Tab. 13 - Presente indicativo di andar in milanese antico

\begin{tabular}{|l|l|l|l|l|l|}
\hline $1 \mathrm{sg}$ & $2 \mathrm{sg}$ & $3 \mathrm{sg}$ & $1 \mathrm{pl}$ & $2 \mathrm{pl}$ & $3 \mathrm{pl}$ \\
\hline vo & ve/vai & va & vam & andei & van \\
\hline
\end{tabular}

Nello specifico, il fenomeno del sincretismo tra le diverse celle di un paradigma può presentare risultati eterogenei: nell'alto limosino nâ 'andare' la generalizzazione del lessema vaděre ha toccato tutto il plurale (tab. 14); in alcune varianti piemontesi è avvenuto un livellamento non verso la $1^{\mathrm{a}}$ pers. pl. , bensì verso la $2^{\mathrm{a}}$ pers. pl. (tab. 15a), ben visibile anche nella distribuzione dell'infisso incoativo (15b), che interessa più da vicino la presente trattazione. Basandosi su HINZELIN, MAIDEN (2018: 284-287) analizza questa incursione su un originario N-pattern ipotizzando l'influenza di un morfoma che viene nominato $D L P$, acronimo di Dark L-pattern: si tratta di uno schema morfomico che si comporta come un L-pattern ma che a differenza di quest'ultimo non espande la cella della $1^{\mathrm{a}}$ pers. $\mathrm{sg}$. del presente indicativo, bensì quella della $3^{\mathrm{a}}$ pers. pl. dello stesso tempo verbale. Una simile affermazione è stata comprovata diacronicamente per l'evoluzione intraparadigmatica nei verbi francesi del tipo prendre 'prendere', dove l'attuale forma della $3^{\text {a }}$ pers. pl. dell' indicativo presente (prennent / etimologicamente, mentre quelle omofone del singolare (prends - prends - prend, anch'esse pronunciate / questa cella su quelle del singolare, che in origine avevano come base preignalmeno fino al XVIII secolo (ESHER, 2017: 63). Questa sorta di «allomorfia» all'interno dei paradigmi morfomici viene presentata da MAIDEN come una delle variazioni possibili dovute all'intervento di fattori extramorfologici di tipo fonologico o funzionale: «Existing patterns may become subject to systematic disruptions in their distribution that, strikingly, leave them still morphomic, but with a changed paradigmatic domain» (2018: 284):

Tab. 14 - Presente indicativo dell'altolimosino nâ 'andare'

\begin{tabular}{|l|l|l|l|l|l|}
\hline $1 \mathrm{sg}$ & $2 \mathrm{sg}$ & $3 \mathrm{sg}$ & $1 \mathrm{pl}$ & $2 \mathrm{pl}$ & $3 \mathrm{pl}$ \\
\hline $\mathrm{vau} /$ vo & vâ & vai & van & vâ & van \\
\hline
\end{tabular}

Tab. 15 - Presente indicativo del piemontese indé (Cairo Montenotte) 'andare' (a) e di finì 'finire' (GRIVA 2007: 83-84) (b)

(a)

\begin{tabular}{|l|l|l|l|l|l|}
\hline $1 \mathrm{sg}$ & $2 \mathrm{sg}$ & $3 \mathrm{sg}$ & $1 \mathrm{pl}$ & $2 \mathrm{pl}$ & $3 \mathrm{pl}$ \\
\hline vag & vai & va & induma & vei & van \\
\hline
\end{tabular}


(b)

\begin{tabular}{|l|l|l|l|l|l|}
\hline $1 \mathrm{sg}$ & $2 \mathrm{sg}$ & $3 \mathrm{sg}$ & $1 \mathrm{pl}$ & $2 \mathrm{pl}$ & $3 \mathrm{pl}$ \\
\hline finisso & finisse & finiss & finioma & finisse & finisso \\
\hline
\end{tabular}

Prendendo spunto dall'analisi di HINZELIN, anche nel caso dei verbi brianzoli saremmo quindi difronte a un diffuso processo di take-over, ossia di «occupazione» di una cella di un paradigma da parte di un'altra; il paradigma da un lato tenderebbe per effetto del sincretismo a livellare l'allomorfia, dall'altra cercherebbe di contrastare la presenza di una resistente struttura morfomica del tipo $N$-pattern, in base alla quale l'infisso incoativo - $(e / i) s k$ - si è radicato nella maggior parte dei dialetti italoromanzi oltre alle varianti romance, con cui il brianzolo può aver intrattenuto contatti. Dallo scontro di queste due forze morfologiche, quindi, emergerebbe come risultato la particolare conformazione presente nei verbi del brianzolo analizzati in questo paragrafo. Il sincretismo si manifesta come una forza morfologica piuttosto intensa anche in brianzolo e merita decisamente un' attenzione particolare, poiché si ritrova anche nelle forme del condizionale di cui si discute nel paragrafo successivo.

\section{3. La doppia serie di condizionale semplice}

Il brianzolo, così come altre varianti settentrionali non solo lombarde, possiede due serie complete di condizionale semplice, la prima formata dall'infinito più le forme contratte dell' imperfetto indicativo di habere (tab. 16), la seconda invece che presenta nelle desinenze una /s/ di origine ancora discussa (tab. 17):

Tab. 16-Condizionale semplice (1) di vené 'venire (infinito + habebam, -as...)

\begin{tabular}{|l|l|l|l|l|l|}
\hline $1 \mathrm{sg}$ & $2 \mathrm{sg}$ & $3 \mathrm{sg}$ & $1 \mathrm{pl}$ & $2 \mathrm{pl}$ & $3 \mathrm{pl}$ \\
\hline vegnarìa & vegnarìet & vegnarìa & vegnarìem & vegnarìi & vegnarien \\
\hline
\end{tabular}

Tab. 17 - Condizionale semplice (2) di vené 'venire' (infinito + habuissem, -es...)

\begin{tabular}{|l|l|l|l|l|l|}
\hline $1 \mathrm{sg}$ & $2 \mathrm{sg}$ & $3 \mathrm{sg}$ & $1 \mathrm{pl}$ & $2 \mathrm{pl}$ & $3 \mathrm{pl}$ \\
\hline vegnarés & vegnarésset & vegnarés & vegnaréssem & vegnarésset & vegnaréssen \\
\hline
\end{tabular}

La prima tipologia di condizionale semplice è maggioritaria nella Romània soprattutto occidentale ma ben radicata pure in diversi dialetti italoromanzi, anche in diacronia (ROHLFS, 1968: 343-344); la seconda invece pone alcune questioni non del tutto chiarite riguardo alla sua evoluzione morfologica ed è di questo modello che ci si occuperà più dettagliatamente.

ILIESCU (1995) ritiene che le forme dei dialetti italiani settentrionali aventi una /s/ nelle desinenze mostrino dei legami con il friulano, in cui il condizionale semplice risalirebbe a un processo di formazione dove l'infinito si è unito con le desinenze del congiuntivo piuccheperfetto latino di habere. L'argomentazione di ILIESCU si basa sulla casistica delle diverse strategie perifrastiche che ebbero luogo nel complesso sviluppo del condizionale romanzo come tempo del futuro a partire dal latino tardo e sul fatto che il congiuntivo piuccheperfetto latino era andato a sostituire le forme dell'imperfetto. In seguito a ciò, nel periodo ipotetico latino si 
verificò una ristrutturazione delle opzioni a disposizione dei parlanti per esprimere la realtà o l'irrealtà di un enunciato in cui, tra le altre, era possibile una situazione dove la protasi e l'apodosi utilizzassero entrambe un congiuntivo imperfetto derivato dal piuccheperfetto (del tipo Si habuissem, dedissem = 'se avessi, darei'); ILIESCU ipotizza che su questa base si possa essere formato un modello di periodo ipotetico non attestato dove l'apodosi iniziò a presentare una forma del tipo infinito più congiuntivo piuccheperfetto/imperfetto di habere, origine del condizionale semplice avente una /s/. D'altronde, per quanto attualmente non vi siano testimonianze nelle fonti latine, non è del tutto fuori luogo accettare come probabile che, così come l'imperfetto indicativo di habere, anche l'imperfetto congiuntivo sia potuto entrare a far parte delle perifrasi indicanti i nuovi tempi verbali romanzi (ILIESCU, 1995: 163); in ogni caso, non sembra che al momento vi possa essere una spiegazione alternativa convincente dell'esistenza di forme verbali aventi una /s/ nelle proprie desinenze.

Se si estende in modo forse azzardato la prospettiva di analisi a tutta la Romània, si può riscontrare che l'ipotesi accennata poc'anzi viene discussa da tempo anche nella consistente bibliografia relativa all'evoluzione del condizionale in rumeno (cfr. COENE ET TASMOSKI, 2006). Come ben noto dalle principali compilazioni tradizionali (cfr. ad es. RENZI, 1994: 188-189), le perifrasi latine formate da infinito e forme di habere alla base del futuro romanzo e del condizionale non erano giunte nelle zone periferiche dell' Impero romano (Sardegna, Italia meridionale, Dacia ...), che hanno così avuto spazio per formulare soluzioni alternative. La maggioranza degli studiosi (per quanto le descrizioni dell'evoluzione diacronica non siano sempre concordi) sostiene che il rumeno abbia sviluppato la morfologia del futuro sfruttando processi presenti anche nelle altre lingue non romanze con cui, per via della sua speciale posizione geografica, ha intrattenuto contatti nell'ambito della Sprachbund balcanica. Riducendo di molto questa complessa questione, va qui perlomeno ricordato che nella formazione della perifrasi dacoromanza avente valore di futuro sono attestati storicamente gli interventi di ben tre diversi ausiliari ( $a$ vrea 'volere', a avea 'avere, a fi 'essere') più le forme dell'infinito, del congiuntivo o del gerundio, ognuno dei quali ha avuto un maggiore o minore successo in diacronia (DIMITRESCU, 1978: 314-316). Attualmente il futuro semplice del rumeno standard presenta tre varianti:1) (v)oi face < *volere ind. pres. + infinito, 2) o să fac < vrea 'volere' pres. 3. sg. invariabile + cong. pres. , 3) am să fac < habere ind. pres. + cong. pres. , tutte col significato di 'io farò' Le prime due tipologie aventi come ausiliare il verbo volere mostrano chiari parallelismi con tutte le altre lingue balcaniche, dal bulgaro al greco moderno passando per l'albanese, mentre la seconda coinvolge l'ausiliare habere, in modo simile alle perifrasi dei dialetti italoromanzi meridionali (cfr. nap. aggio a $i<$ habeo ad ire, lett. 'ho da andare', 'andrò') (RoHLFS, 1968: 335-336). A differenza del futuro semplice, il condizionale ha però una sola forma analitica composta da un ausiliare a cui viene aggiunta la base dell'infinito (tab. 18):

Tab. 18 - Condizionale semplice del rumeno a face 'fare'

\begin{tabular}{|l|l|l|l|l|l|}
\hline $1 \mathrm{sg}$ & $2 \mathrm{sg}$ & $3 \mathrm{sg}$ & $1 \mathrm{pl}$ & $2 \mathrm{pl}$ & $3 \mathrm{pl}$ \\
\hline aș face & ai face & ar face & am face & ați face & ar face \\
\hline
\end{tabular}


L'accesa discussione sull'origine dell'ausiliare che forma la perifrasi indicante il condizionale semplice nelle lingue dacoromanze è molto complessa e dibattuta (per un contributo articolato e recente, cfr. ZAFIU, 2017). Sintetizzando le posizioni in campo, si può brevemente ricordare che da una parte c'è chi argomenta a favore di un'evoluzione dell'imperfetto di *volere esattamente come avvenuto nel caso dei futuri semplici di tipo «panbalcanico» visto più sopra; altri sottolineano il possibile ruolo dell'imperfetto indicativo latino dell'ausiliare ESSE soprattutto per giustificare le forme delle terze persone (??era facere > ar face); altri ancora, in particolar modo gli autori a cavallo tra XIX e XX secolo come Meyer-Lübke o Rosetti, invece ritengono che l'ausiliare derivi dal congiuntivo piuccheperfetto latino di habere (habuissem, -es, ...), come appunto nel caso dell'ipotesi avanzata per il brianzolo e per gli altri dialetti italiani settentrionali, soprattutto per giustificare l'ausiliare della prima persona (aș face); infine vi è anche chi ipotizza una contaminazione tra le diverse opzioni. Senza entrare nel merito di questo dibattito, che per quanto interessante rischia di uscire dai limiti di un contributo limitato a un dialetto italoromanzo, ritengo tuttavia che possa essere significativo, seppur con le dovute differenze e cautele rispetto alla situazione del brianzolo, osservare che la dinamica diacronica relativa alla formazione del condizionale rumeno mostra un percorso contorto e non univoco, probabilmente utile per ricostruire quanto avvenuto anche nelle fasi storiche non attestate dei dialetti italiani settentrionali. Anche in questo senso uno spunto proviene dallo spoglio riportato da DOMOKOS effettuato sulle opere in volgare di Bonvesin de la Riva (2007: 268-269), in cui sono presenti ben tre diverse forme di condizionale: una di tipo maggioritario romanzo (infinito + imperfetto di habere: porria < *potere + habebam 'io potrei'), una di tipo toscano (infinito + perfetto di habere: porev/poreve/porreve $<$ *potere + *hebui 'io potrei') e una analitica (perfetto di habere + infinito: ev offende $<*$ hebui + offendere 'io offenderei').

La contaminazione tra due o addirittura tre paradigmi morfologicamente differenti è abbastanza diffusa nei dialetti italoromanzi in diverse aree anche al di fuori della Lombardia (ILIESCU, 1995: 160-161), e come osservato nel paragrafo precedente a quanto pare non è sconosciuta, almeno in linea di principio, ad altre lingue romanze. ROHLFS (1968: 341-343) attribuisce la contaminazione nei dialetti italiani a un effetto dell' analogia tra congiuntivo imperfetto e condizionale (vedeste : vedreste $=$ vedessimo $:$ vedressimo , affermando persino che un paradigma unico per il condizionale è un caso piuttosto raro. Anche nel brianzolo l'interferenza tra le due forme di condizionale semplice viene parzialmente attestata da alcuni informatori, ma a causa del livello ancora frammentario dei dati finora raccolti, non è possibile al momento delinearne una tipologia. Tuttavia, se in futuro verrà confermata questa ipotesi, uno spunto ulteriore di ricerca potrà orientarsi a quanto è ben attestato in molte varietà dialettali anche nelle loro fasi storiche o persino nell'italiano letterario del Cinquecento di autori come Baldassar Castiglione (ROHLFS, 1968: 344), in cui si manifesta una distribuzione morfomica tra due paradigmi del condizionale di origine diversa e definita da MAIDEN (2018: 289-291) come E-pattern, ossia una variante contratta di $N$-pattern in cui viene esclusa la 2. 
pers. sg. , tipica soprattutto per i perfetti forti italoromanzi (del tipo dissi-dicestidisse...).

Oltre all'aspetto morfologico, spunti per l'analisi del condizionale semplice in brianzolo derivano anche dall'osservazione del suo uso a livello sintattico e pragmatico, che in questa sede verrà solo accennato. Nel periodo ipotetico del secondo tipo, il condizionale con le desinenze in /s/ può svolgere esclusivamente la funzione di protasi, cosa che il condizionale formato da infinito e desinenze dell'imperfetto di habere invece può fare in alternativa a quella di apodosi (5):

(5) Se'l vegnarés anca lü, gh'el dumandarìa

Se 'venire' - COND. PRES. (b). 3SG. anche lui, glielo 'domandare' - COND. PRES. (a). 1SG.

Se'l vegnarìa anca lü, gh'el dumandarìa

Se 'venire' - COND. PRES. (a)3SG. anche lui, glielo 'domandare' - COND. PRES(a). 1SG.

'Se venisse anche lui, glielo domanderei'

A livello pragmatico, secondo alcuni informatori è possibile anche osservare una diversa forza illocutoria dei due tipi di condizionale quando vengono utilizzati in una frase semplice (6-7):

(6) Carletu, te vegnarìes a vangò la vigna?

Carletto, 'venire' - COND. PRES. (a). 2SG. a vangare la vigna?

Carletto, verresti a vangare la vigna?

(7) Carletu, te vegnaresset a vangò la vigna?

Carletto, 'venire' - COND. PRES. (b). 2SG. a vangare la vigna?

Carletto, (non è che per caso) verresti (gentilmente) a vangare la vigna?

\section{Conclusioni}

In seguito alla panoramica effettuata sui dati relativi ad alcuni aspetti della morfologia del verbo brianzolo, è certamente possibile perlomeno riconoscere che anche questa parlata partecipa pienamente alla complessità ancora non del tutto esplorata dei dialetti italoromanzi. I tre fenomeni analizzati meritano di essere ulteriormente approfonditi basandosi, oltre che su un'indagine appropriata dei dati, su ipotesi formulabili sulla base dei parallelismi riscontrabili in altre varianti romanze, contigue e non, in primis nel milanese ma anche nelle parlate del Canton Ticino e di altre varianti settentrionali. In particolar modo ciò che in questo articolo, in mancanza di altre definizioni, è stato etichettato come «imperativo futuro» (3. 1) potrebbe avere un corrispettivo in ciò che viene segnalato nel romancio dell'Engadina superiore (SCHEITLIN, 1980: 81) come «futuro secondo», un tempo formato dall'infinito con le desinenze provenienti dal congiuntivo presente di habere e che pare esprimere incertezza o desiderio, invece di avere un valore iussivo come nel caso del dialetto brianzolo. Il sincretismo in atto nei paradigmi aventi un infisso incoativo (3.2) indica probabilmente che la tendenza a eliminare l'allomorfia, fenomeno abbastanza tipico nella morfologia verbale romanza, si scontra con la tendenza opposta a mantenere un contrasto tra la $2^{\mathrm{a}}$ pers. pl. e il resto delle celle; oltre a dover spiegare il motivo per cui è proprio questa cella del paradigma a distinguersi dalle altre (forse a causa della sua bassa frequenza di uso), 
la questione in futuro sarà valutare se e quanto a lungo una simile situazione resisterà e quale direzione prenderà la conformazione di questi paradigmi, se verso un completo livellamento analogico o verso una conformazione da $N$-pattern. Per quanto riguarda invece la doppia serie di condizionali semplici (3. 3), il rapporto tra le due tipologie di paradigmi e gli usi sintattico-pragmatici potrebbero aprire nuovi orizzonti sia nella 'morfomicità' di alcune distribuzioni miste che nel ruolo svolto da altri fattori, quali la modalità e l'Aktionsart. In questa prospettiva, sembra evidente che anche il dialetto brianzolo mostra di essere sensibile a dinamiche di «morfologia autonoma» che hanno il solo difetto di non poter essere studiate in diacronia a causa della tarda e scarsa tradizione scritta.

\section{BIBLIOGRAFIA}

ARONOFF Mark (1976), Word Formation in Generative Grammar, Cambridge, Mass., MIT Press.

ARONOFF Mark (1994), Morphology by itself: Stems and inflectional classes, Cambridge, Mass., MIT Press.

BENINCÀ Paola, PARRY Mair, PESCARINI Diego (2016), The dialects of northern Italy, in: LEDGEWAY Adam, MAIDEN Martin (a cura di), The Oxford guide to the Romance languages, Oxford, Oxford University Press, p. 185-205.

BIONDELli Bernardino (1853), Saggio sui dialetti gallo-italici, Milano, G. Benardoni.

BONFADINI Giovanni (2010a), I dialetti, in: BRESSAN Edoardo (ed. ), Storia della Brianza, vol. V, 'Le culture popolari', Oggiono-Lecco, Cattaneo Editore, p. 218-243.

BONFADINI Giovanni (2010b), Lombardi, dialetti, in: SiMONE Raffaele (a cura di), Enciclopedia dell'italiano, Roma, Istituto dell'Enciclopedia Italiana, p. 840843. Disponibile anche in http://www. treccani. it/enciclopedia/dialettilombardi_(Enciclopedia-dell'Italiano)/ [consultato il 25-9-2019]

BYBEE Joan L. (2007), Frequency of use and the organization of language, Oxford, Oxford University Press.

ChERUBINI Francesco (1856), Vocabolario Milanese-Italiano, vol. V: Sopragiunta. Nozioni filologiche intorno al dialetto milanese. Saggio d'osservazioni su l'idioma brianzuolo, suddialetto del milanese, Milano, Soc. tip. de' Classici italiani.

CoENE Martine, TASMOSKI Liliane (2006), On the Balkan-Slavic origins of the Romanian conditional, Revue roumaine de linguistique 51/2, p. 321-340.

CORBETT Greville (2005), The canonical approach in typology, in: FRAJZYNGIER Zygmunt, HodGES Adam, RooD David S. (a cura di), Linguistic Diversity and Language Theories), Philadelphia, John Benjamins Publishing Company, p. 2549.

CorbetT Greville G. (2016) Morphomic splits, in: Luís Ana R. , BERMúdeZOTERO Ricardo (a cura di), The morphome debate, Oxford, Oxford University Press, p. 64-88. 
CROLA Pierluigi (2005), Il lombardo occidentale prealpino (Como, Lecco, Sondrio, Varese), in: RoGNONI Andrea (a cura di), Grammatica dei dialetti della Lombardia, Milano, Mondadori, p. 93-137.

DiMITRESCU Florica (a cura di) (1978), Istoria limbii române: Fonetică, morfosintaxă, lexic, Bucureşti, Editura Didactică şi pedagogică.

DOMOKOS György (2007), La morfologia verbale del milanese antico di Bonvesin de la Riva, Verbum Analecta Neolatina IX/2, p. 261-277.

ESHER Louise. (2017). Morphome death and transfiguration in the history of French. Journal of Linguistics 53, 51-84.

FLORICIC Franck, MOLINU Lucia (2012), Romance Monosyllabic Imperatives and Markedness, in: STOLZ Thomas, NAU Nicole, STROH Cornelia (a cura di), Monosyllables: from phonology to typology, Berlin, Akademie Verlag, p. 149172.

GrIVA Guido (2007), Grammatica della lingua piemontese, Torino, Viglongo.

HINZELIN Marc-Olivier (2011), Syncretism and Suppletion in Gallo-Romance Verb Paradigms, in: MAIDEN Martin, SMITH John Charles, GoldBACH Maria, HINZELIN Marc-Olivier (a cura di), p. 287-310.

ILIESCU Maria (1995), Le conditionnel frioulan, in: Scritti di linguistica e dialettologia in onore di Giuseppe Francescato, Trieste, Edizioni Ricerche, p. 159-165.

LOPORCARO Michele (2009), Profilo linguistico dei dialetti italiani, Roma, Laterza. MAIDEN Martin (2007), On the morphology of Italo-Romance Imperative, in: BENTLEy Delia, LEDGEWAY Adam (a cura di), Sui dialetti italoromanzi: saggi in onore di Nigel B. Vincent, Norfolk, Biddles, p. 148-164.

MAIDEN Martin (2018) The romance verb: morphomic structure and diachrony, Oxford, Oxford University Press.

MAIDEN Martin, CRUSCHINA Silvio, SMITH John Charles (a cura di) (2013), The boundaries of pure morphology: diachronic and synchronic perspectives, Oxford, Oxford University Press.

MAIDEN Martin, SMITH John Charles, GOLdBACH Maria, HINZELIN Marc-Olivier (a cura di) (2011), Morphological autonomy. Perspectives from Romance Inflectional Morphology, Oxford, Oxford University Press.

MAIDEN Martin, SWEARING Andrew, O'NeILL Paul (2009), Imperative morphology in diachrony. Evidence from Romance languages, in: DUFRESNE Monique, DuPUIS Fernand, VOCAJ Etleva (a cura di), Historical linguistics 2007: Selected papers from the 18th international conference on historical linguistics, Montreal, 6-11 August 2007, Philadelphia, PA, John Benjamins Publishing Company, p. 99-108.

MEuL Claire (2010), The intra-paradigmatic distribution of the infix -I/ESC- from Latin to Modern Romance: Morphomic patterning and beyond, Morphology 20, p. 1-40.

PIRRELli Vito (2000), Paradigmi in Morfologia. Un approccio interdisciplinare alla flessione verbale dell'italiano, Pisa-Roma, Istituti editoriali e poligrafici internazionali. 
PIRRELli Vito, BATTISTA Marco (2000), The paradigmatic dimension of stem allomorphy in Italian verb inflection, Italian Journal of Linguistics 12 (2), p. 307-380.

RENZI Lorenzo (1994), Nuova introduzione alla filologia romanza, Bologna, Il Mulino.

RIPAMONTI Fabio (2017), Il suppletivismo verbale romanzo in diacronia. České Budějovice, Episteme.

RiVA Virginio (1987), Le origini della Brianza, Merate, Bertoni.

ROHLFS Gerhard (1968), Grammatica storica dell'italiano e dei suoi dialetti. Morfologia, Torino, Einaudi.

Rossini Giorgio (1975), Capitoli di morfologia e sintassi del dialetto cremonese, Firenze, La Nuova Italia.

SCHEITLIN Walter (1980), Il pled puter. Grammatica ladina d'Engiadin'ota cun lecziuns e register da pleds / Grammatik in Puter (Oberengadin) mit Lektionen und Wörterverzeichnis, Samedan, Uniun dals Grischs.

STUMP Gregory T. (2006), Heteroclisis and paradigm linkage. Language 82, p. 279322.

SWEARING Andrew (2011), The Romance Imperative, Irregular Morphology, Sincretism, and the Morphome, in: MAIDEN Martin, SMITH John Charles, GolDBACH Maria, HINZELIN Marc-Olivier (a cura di), p. 119-134.

THOMPSON Regina Casey (2015), The Erosion of Brianzolo: The Impact of Standard Italian on a Dialect and a Cultural Identity, College of William \& Mary Undergraduate Honors Theses Paper 206. Disponibile anche in https://scholarworks. wm. edu/cgi/viewcontent. cgi?referer=https://it. wikipedia. org/\&httpsredir $=1 \&$ article $=1210 \&$ context $=$ honorstheses [consultato il 25-9-2019]

VINCENT Nigel (2014), Similarity and diversity in the evolution of Italo-Romance morphosyntax, in: BENINCÀ Paola, LEDGEWAY Adam, VINCENT Nigel (a cura di), Diachrony and dialects. Grammatical Change in the Dialects of Italy, Oxford, Oxford University Press, p. 1-21.

ZAFIU Rodica (2017), The auxiliary of the Romanian conditional: Semantic and functional arguments concerning the reconstruction of a disputed grammaticalization process, Diacronia 6, p. 1-20. 